\title{
Procesos de construcción identitaria y desafíos del antiesencialismo analítico
}

\author{
Rita Sobczyk* \\ Rosa Soriano-Miras** \\ Andrés Caballero-Calvo*
}

\section{Resumen}

La comprensión de las tensiones sociales y políticas actuales genera un renovado interés en el estudio de los procesos de construcción identitaria. Sin embargo, el concepto de "identidad", pese a su gran presencia en los discursos públicos, sigue siendo afectado por una profunda ambigüedad definitoria que limita su utilidad analítica y dificulta la transferencia de conocimiento de las investigaciones científicas que pudieran contribuir a debates informados en el ámbito público. El presente artículo, en base a la revisión de estudios existentes, aboga por la conceptualización de la identidad como un proceso multidimensional. La elaboración teórica sobre los mecanismos de construcción identitaria lleva a plantear cinco precauciones principales a considerar a la hora establecer relaciones entre las categorías identitarias y las pautas sociales. Se realiza una reflexión sobre la necesidad de combinar el antiesencialismo analítico con el estudio de los esencialismos cotidianos y sus implicaciones.

Palabras-clave: fronteras sociales, esencialismo, categorización, constructivismo.

\footnotetext{
* Universidad del Norte, Barranquilla, Colombia.

** Universidad de Granada, Granada, España.
} 


\section{Identity construction processes and challenges of analytical anti-essentialism}

\section{Abstract}

The understanding of present social and political tensions generates a renewed interest in the study of identity construction processes. However, the concept of "identity", despite its wide presence in the public discourses, remains profoundly affected by ambiguity in terms of its definition. This limits its analytical usage and hinders the dissemination of the findings of scientific research that could contribute to creating informed debates in the public domain. The present paper, based on the review of existing studies, argues for a conceptualization of identity that defines it as a multidimensional process. The theoretical discussion concerning the main mechanisms of identity construction leads us to propose five precautions which should be taken into account when analysing the relation between identity categories and social patterns. Finally, we reflect on the necessity of combining analytical anti-essentialism with the study of everyday essentialisms and their implications.

Keywords: social boundaries, essentialism, categorization, constructivism.

\section{Introducción}

- A qué nos referimos cuando hablamos sobre "identidad"? Esta pregunta 2 vuelve a adquirir especial relevancia en el presente contexto de fomento de las reivindicaciones nacionalistas en países tan diversos como Suecia, Estados Unidos, Brasil o Rusia. Los actuales discursos anti-inmigración, que adquieren tintes xenófobas y racistas, convierten el análisis de los procesos de construcción identitaria en un ámbito de investigación urgente. Sin embargo, el concepto de "identidad" es comprendido y definido de maneras tan diversas que su utilidad analítica ha sido cuestionada. El ya clásico texto de Brubaker y Cooper (2001) destaca que este único término ha sido aplicado para referirse a fenómenos sociales profundamente heterogéneos. La ambigüedad del término, así como su fuerte carga esencialista, indujo a estos autores a rechazar el concepto por completo. En el presente artículo, siguiendo las ideas de Henry Hale (2004), se argumenta que, en lugar de 
abandonar el término, la identidad debería ser analizada como un proceso multidimensional. El texto, inspirado principalmente en las aportaciones de Henry Hale (2004; Hale; Shevel; Onuch, 2018), Andreas Wimmer (2008a; 2013; 2014), Rogers Brubaker (2009; 2012; Brubaker; Cooper, 2001; Brubaker; Loveman; Stamatov, 2004) y sus colaboradores, ofrece una reflexión teórica sobre las principales dificultades definitorias del concepto.

La problemática de la identidad es compleja y su estudio requiere de enfoques sensibles a factores contextuales que intervienen en los procesos de construcción de la idea del grupo en poblaciones específicas. Sin embargo, consideramos que los debates actuales requieren de un mayor nivel de sistematización. En este sentido, el presente artículo complementa los estudios de otros investigadores, como por ejemplo los ampliamente citados textos de Andreas Wimmer (2008a, 2008b, 2013, 2014) sobre las fronteras étnicas, que defienden la necesidad de sistematizar el conocimiento existente sobre la identidad y las fronteras sociales.

En el primer apartado, partiendo de una revisión de las investigaciones existentes, se debate la utilidad analítica del concepto. Seguidamente, se analizan los principales mecanismos que intervienen en los procesos de construcción identitaria. Se pone un énfasis especial en los elementos de la realidad que llevan al surgimiento de categorías identitarias de alta relevancia social, que pueden movilizar la acción colectiva y fomentar las conexiones intragrupales. La definición de identidad defendida nos lleva, en el último apartado, a debatir cinco precauciones fundamentales que se deberían considerar a la hora de analizar la relación entre las categorías identitarias y las pautas de comportamiento de las personas etiquetadas con estas categorías.

\section{Identidad como un proceso multidimensional}

La raíz latina del concepto "identidad", idem, se traduce como "lo mismo", transmitiendo la idea de igualdad o similitud (Jenkins, 2008). En la literatura existente se distingue entre la identidad personal y la identidad 
social. La primera se basa en atributos personales, mientras que la segunda se apoya en la pertenencia a grupos (Gómez; Vázquez, 2015). Pese a que el concepto "identidad" se ha popularizado sobre todo desde los años 60, otras nociones emparentadas constituyen parte intrínseca de la historia de la humanidad y de la reflexión en las ciencias sociales. El estudio de la identidad social es heredero de los teóricos clásicos, vinculándose con términos como la "conciencia de comunidad", de Weber, o la "conciencia colectiva", de Durkheim. La relevancia del concepto en la investigación actual queda plasmada en los estudios de muchos de los investigadores más reconocidos de las últimas décadas, como Castells (2004), Bauman (2013), Tilly (2015) o Hall (2014).

Siguiendo la definición y las aportaciones teóricas de Henry Hale (2004), abogamos por la necesidad de conceptualizar la identidad personal como el conjunto de puntos de referencia vinculados entre sí, construidos socialmente y cambiantes en cuanto a su significado y relevancia, que sirven para la auto-definición personal, estableciendo una relación entre el individuo y las demás personas y orientando potencialmente el comportamiento. En cada momento dado la identidad está formada por puntos de referencia de baja y de alta relevancia social. Un punto de referencia de alta relevancia social influye profundamente en la forma en la que se interpreta la realidad ${ }^{1}$. Por el contrario, los referentes de baja relevancia tienen escasa repercusión en la vida cotidiana.

Los puntos de referencia se asocian con categorías identitarias. Entre las más frecuentemente utilizadas destacan las que tienen que ver con edad, género, profesión, religión o nacionalidad. Las categorías identitarias pueden ser clasificadas siguiendo distintas tipologías. Sirvan de ilustración los ejemplos aportados en el texto de Escobar, Revilla y Sánchez-Sierra (2018).

'El concepto de "saliencia de la identidad", muy bien asentando en la literatura existente (Stets; Burke, 2000), se relaciona estrechamente con la noción de alta relevancia utilizada en el presente trabajo. Si bien ambos términos permiten captar el proceso de activación de los referentes identitarios, consideramos que el segundo expresa mejor la idea de que las identidades sociales se ubican en un continuum y que su importancia, tanto a nivel perceptual como conductual, puede variar en un amplio espectro en función del contexto. 
Los individuos pueden definirse en términos, entre otros, de pertenencia a grupos sociales amplios asociados a territorio, nación, etnia, clase social, género, edad, religión, ideología, convicciones o lenguaje (hispanohablante, liberal, colombiano, hombre, viejo, musulmán, tutsi, pobre), humanidad (humano, ciudadano del mundo), actividades (médico, estudiante, activista), rol en la familia (esposo, hija), personalidad (tranquilo, colérico), aspecto físico (alto, moreno), emociones (feliz, ansioso), aspiraciones (futuro director), propiedad (propietario de un apartamento, finca), experiencias vividas (superviviente de un accidente), creencias (creyente en el destino, suerte) u ocio (ciclista, aficionado al cine). Al definir las categorías se establece un límite entre quién pertenece a ellas y quién no. De ahí que la inclusión de ciertos individuos en una categoría inevitablemente conlleve la exclusión de otros, asociándose con frecuencia con la distribución desigual de recursos económicos, prestigio social o un diferenciado acceso al poder.

La combinación de múltiples puntos de referencias es lo que hace único a cada individuo. En este sentido, la identidad nos hace singulares, permitiendo diferenciarnos de los demás. Sin embargo, no nos podemos olvidar, al mismo tiempo, que las pertenencias particulares nos unen con las personas con las que compartimos las mismas categorías identitarias, si bien el significado y relevancia de las categorías identitarias puede variar de un individuo a otro y de un contexto a otro.

Las categorías identitarias son utilizadas para simplificar la complejidad de la realidad que nos rodea. La categorización es "el proceso por el que se ordena el entorno mediante categorías, tales como grupos de personas, objetos y hechos que tengan alguna característica similar o equivalente entre sí, que sea relevante para las acciones, intenciones o actitudes de los individuos" (Tajfel, 1984, p. 160). Así, las personas simplifican el mundo social haciendo generalizaciones. Las categorías identitarias constituyen una herramienta que nos permite ubicarnos en la telaraña de relaciones sociales. Al encontrarnos con personas extrañas utilizamos las categorías identitarias para hacernos una idea de qué podemos potencialmente esperar de ellas. 
Ordenamos la realidad en categorías que, a su vez, pueden orientar nuestro comportamiento y nuestras formas de relacionarnos. Por tanto, el análisis de la identidad debe tener en cuenta tanto los discursos como la conducta, abordando no solo lo que se dice, sino también lo que se hace a raíz de los procesos de categorización. En esta línea, Lamont y Molnár (2002) distinguen entre los aspectos simbólicos y sociales de las fronteras sociales. El proceso de categorización conlleva la división intersubjetiva de la población en grupos en base a la percepción de similitudes y diferencias. Esto, a su vez, suele generar un trato diferenciado de personas clasificadas en distintas categorías identitarias. Las categorías identitarias pueden fomentar la relevancia de ciertos tipos de relaciones sociales (por ejemplo, llevando a patrones de endogamia o trato preferencial de los miembros del endogrupo en los procesos de contratación) o repercutiendo en otras prácticas culturales (por ejemplo, asociándose al uso de una lengua común).

Deberíamos aclarar que el hecho de compartir ciertas pautas de comportamiento no necesariamente implica que surja el sentimiento de pertenencia grupal. Por ejemplo, un ciudadano de un Estado puede compartir la nacionalidad con otros habitantes del territorio estatal, hablar el mismo idioma y relacionarse en su vida cotidiana sobre todo con sus compatriotas, pero esto no necesariamente significa que la pertenencia a la nación sea algo relevante para su auto-definición.

De igual manera, la existencia de discursos que evocan ciertas categorías identitarias no tiene por qué traducirse en el surgimiento de pautas de comportamiento colectivas. En el ámbito de la etnicidad, por ejemplo, pueden surgir discursos políticos que abogan por la unidad colectiva, pero esto no necesariamente se plasma en un comportamiento real de las personas etiquetadas con la categoría étnica. Además, la identidad puede ser profundamente situacional. Ya que las categorías identitarias se asocian con expectativas en cuanto a la conducta, en la vida cotidiana frecuentemente actuamos, muchas veces de manera improvisada, intentando ajustarnos al imaginario asociado a nuestras diversas pertenencias (Goffman, 2001). Sin 
embargo, también podemos cuestionar, matizar o incluso rechazar las ideas hegemónicas sobre el comportamiento asociado a categorías particulares.

Las deficiencias en la definición del concepto de identidad tienen que ver, en gran medida, con la inadecuada comprensión de las posturas primordialista y constructivista, que obstaculiza la apreciación de las aportaciones teóricas de cada una de estas perspectivas (Hale, 2004). La simplificación desmesurada de la postura primordialista lleva a la idea de que algunas dimensiones identitarias son fijas, estáticas, inmutables. Sin embargo, la mayor parte de los científicos en la actualidad, incluidos los primordialistas, coincide en que las identidades son construidas socialmente y que se forjan a raíz de la socialización.

Si los investigadores coinciden de manera mayoritaria en que la identidad es construida, el debate científico debería centrarse sobre todo en la variación que puede afectar a los diferentes tipos de pertenencias, tanto en cuanto a su significado como en cuanto a su relevancia. El estudio de la identidad se enfocaría así en preguntas como: ¿cómo se construyen las categorías identitarias?, ¿qué actores sociales intervienen en este proceso y para qué lo hacen?, ¿̇e qué poder disponen?, ¿cómo las categorías influyen en nuestras relaciones y prácticas culturales?, ¿cuál es su nivel de resistencia al cambio? Se debería tener en cuenta, además, tanto el impacto de las estructuras sociales como de la agencia humana, ya que los individuos no solo interiorizamos quiénes somos sino que, de manera activa, podemos moldear nuestras pertenencias. Llevando estos supuestos, por ejemplo, al campo del estudio del nacionalismo, deberíamos preguntarnos en qué circunstancias y por qué las personas empiezan a interpretar el mundo en términos nacionalistas en lugar de utilizar otras categorías. En el siguiente apartado analizaremos cómo se construyen las categorías identitarias colectivas, poniendo especial énfasis en aquellas que tienen el potencial de adquirir muy alta relevancia social y llevar al surgimiento de fronteras sociales "claras", utilizando la terminología de Alba (2005). 


\section{Procesos de construcción identitaria}

Las categorías identitarias colectivas, es decir, aquellas que sirven para crear la idea del grupo, pueden ser construidas internamente y externamente. El pensamiento de Mead (2009 [1934]) fue clave para enfocar el estudio de la identidad hacia la relación existente entre las identificaciones externas y la auto-definición personal. Los discursos y prácticas de inclusión y exclusión realizadas por diversos actores sociales pueden influir en cómo se definen las personas a nivel identitario. Deberíamos preguntarnos, por tanto, por el proceso de identificación que, partiendo del carácter reflexivo del ser humano, permite categorizar y nombrar tanto a uno mismo como a los demás. A nivel colectivo, tanto el endogrupo como el exogrupo pueden involucrarse en la creación de la idea del grupo, generando categorías identitarias y fronteras sociales asociadas a estas. En este proceso, la clave es la percepción de similitud intragrupal y diferencia con los demás. Se destaca lo que no somos para crear la idea de quiénes somos. Sin embargo, no es suficiente con generar una categoría propia. Esta tiene que ser aceptada por los demás, por lo que el análisis de la identidad requiere del estudio de las relaciones, conflictos y tensiones entre grupos.

Al mismo tiempo, incluso en el seno de un colectivo particular, la auto-identificación puede quedar sujeta a choques y negociación. El poder de las élites del endogrupo puede ser clave en la delimitación de las fronteras colectivas y de los criterios de pertenencia. Sirva de ilustración el caso del genocidio de Ruanda de 1994, que, según Uvin (1999), se ha asociado con la consolidación de las categorías étnicas. Las fronteras sociales anteriores eran, sobre todo, regionales y sociales, pero no étnicas. La promoción del discurso del conflicto entre Hutus y Tutsi y su inclusión en la política sirvieron para legitimar el control del estado por parte de las élites y obstaculizar la democratización, si bien el legado del colonialismo belga también contribuyó a esta realidad.

La cuestión del poder es clave para entender cómo surgen las categorías identitarias colectivas y las fronteras sociales. El análisis de las relaciones 
de poder es necesario para poder comprender qué actores sociales tienen mayor probabilidad de imponer su definición de la realidad social. En este sentido, algunos investigadores recomiendan hacer una distinción entre "quién hace" y "quién padece" las categorías identitarias existentes (Olmos, 2009). Es importante enfatizar que normalmente tanto el endogrupo como los actores sociales externos suelen participar en el proceso de "hacer" las categorías identitarias. Sin embargo, solo algunas de estas categorías llegarán a adquirir peso en la estructuración de las relaciones sociales.

La población que disfruta de un mayor acceso al poder suele tener una mayor probabilidad de crear categorías identitarias que se convierten en las dominantes y que, por tanto, tendrán una mayor repercusión en la vida cotidiana. Las etiquetas creadas de esta manera pueden servir para legitimar las relaciones de dominación sobre grupos minoritarios. Así, la libertad de construir nuestras identidades de manera reflexiva (Giddens, 1991) puede verse profundamente obstaculizada.

Los procesos de categorización se vinculan estrechamente con las desigualdades sociales. Las jerarquías entre grupos son el fruto de la atribución de juicios de valor a los discursos de la diferencia. La definición de "otro" grupo en términos negativos puede servir para legitimar su discriminación y el trato preferencial para los miembros del endogrupo. Las jerarquías sociales, fundamentadas en oposiciones binarias, suelen hacerse en base a las identificaciones realizadas por los actores sociales dominantes, permitiendo justificar de esta manera su posición privilegiada. Sirvan de ilustración mecanismos que actúan en el caso de las divisiones étnicas, raciales y nacionales para crear, entre otros, la imagen de atraso de las minorías. Baumann y Gingrich (2006) analizan, por ejemplo, el proceso de orientalización. Este se fundamenta, por una parte, en la construcción de la idea de los "otros" como un reflejo invertido de "nosotros" ("lo que es bueno en nosotros falta en ellos"). Por otra, la orientalización puede plasmarse en la imagen romantizada de los "otros" como personas arraigadas en el pasado ("lo que falta en nosotros está (aún) presente en ellos") (Baumann; Gingrich, 2006, p. x). 
Entre los actores sociales de mayor influencia a la hora de imponer las categorías identitarias se encuentran los Estados. En sus prácticas oficiales de clasificar y contabilizar la población pueden acabar construyendo lo que aparentemente describen. La época colonial ha sido especialmente bien estudiada en este aspecto. Las sociedades conquistadas eran clasificadas en categorías ordenadas de manera jerárquica. Algunas identificaciones creadas por los imperios coloniales llegaron a traducirse en categorías de auto-definición. Sirva de ilustración el caso de la categoría "Yoruba". A pesar de que hoy denota al grupo étnico dominante en la Nigeria actual, se trata de una etiqueta que surge en el contexto colonial del siglo XIX (Peel, 2000).

Los seres humanos definimos las categorías que utilizamos y establecemos fronteras entre ellas. Este supuesto tiene implicaciones cruciales para la comprensión de las desigualdades sociales. Tal y como afirma Olmos (2009, p. 57), "que una situación social de desigualdad provocada por relaciones de alteridad sea una construcción social, significa que es evitable y que puede ser de otra manera. Es decir, que no posee ninguna fuerza intrínseca natural que nos impida denunciar y cambiar esa situación". Pensar las categorías identitarias como un producto humano induce, además, a analizar los actores sociales que se involucran en su construcción.

Pese a las limitaciones estructurales, los individuos pueden intentar evadir o cuestionar las etiquetas externas. Sirva de ejemplo la migración de profesionales de Este a Oeste de Europa, donde a veces se intenta compartir la menor cantidad de información posible sobre su origen para evitar ser vistos como empobrecidos europeos del Este (Csedö, 2010).

Rogers Brubaker ha sido uno de los autores más influyentes en cuanto a la deconstrucción de algunos términos clave en ciencias sociales. En sus estudios, ha cuestionado los usos de conceptos como el de "grupo étnico" (2002), "diáspora" (2005) o "identidad" (Brubaker; Cooper, 2001). Su crítica se ha enfocado principalmente en el impacto del esencialismo en la investigación. Reconociendo esta visión, defendemos sin embargo que la deconstrucción debe tener sus límites. 
La identidad, los grupos étnicos y las diásporas existen en la medida en que los individuos se involucran a nivel colectivo en procesos de construcción de la diferencia. Estos procesos, a su vez, repercuten en nuestro comportamiento. Nuestros sistemas de categorización son fuertemente consecuenciales. Por ejemplo, las categorías identitarias pueden asociarse con patrones de discriminación, convirtiendo nuestra forma de ver el mundo social en realidades muy tangibles. Los límites grupales existen aunque son porosos, cambiantes y definidos de maneras distintas por diferentes actores sociales.

Las categorías identitarias de alta relevancia social, en su forma más extrema, conllevan el desarrollo de sentido de pertenencia exclusivista al grupo y la separación con los demás. Esto puede darse incluso en el caso de grupos tan numerosos como las naciones, implicando el surgimiento de comunidades fuertemente imaginadas (Anderson, 1993) y sentidas.

La vinculación con el endogrupo, siguiendo la teoría de la identidad social, es acompañada por la despersonalización, que conlleva la percepción de uno mismo no como un individuo único sino como componente de categorías sociales más amplias (Stets; Burke, 2000). Los grupos que llegan a formarse de manera exitosa satisfacen dos necesidades opuestas de los individuos (Brewer; Silver, 2000). Por un lado, la búsqueda de la identidad social está motivada por la necesidad de pertenecer y asimilarse, pero, al mismo tiempo, esta tendencia se ve acompañada por la necesidad de diferenciarse de los demás.

Una categoría identitaria se convierte en relevante a nivel colectivo cuando una población se define a sí misma y es definida por los demás como un grupo. Si a la vez los individuos alinean sus propias preocupaciones e intereses con los del endogrupo, están dispuestos a defender sus símbolos, favorecen a los miembros del grupo propio y discriminan a los demás, podemos hablar sobre categorías identitarias de muy alta relevancia social. En el contexto migratorio, por ejemplo, este tipo de fronteras surge cuando la distinción entre la población dominante y las poblaciones de origen 
inmigrante se ve institucionalizada en diversos ámbitos y se asocia con asimetrías en el acceso al poder y a los recursos económicos.

Las dimensiones identitarias de muy alta relevancia social surgen en momentos y periodos específicos de la historia. Por ejemplo, la pertenencia nacional en ciertas circunstancias puede ser fundamental para movilizar la acción colectiva y generar solidaridad intragrupal, mientras que en otras puede ser vista como algo totalmente irrelevante. Los puntos de referencia no constituyen una característica estable e intrínseca de los grupos humanos. Suelen aparecer en periodos de crisis, incertidumbre y profundas transformaciones sociales. Los conflictos bélicos, factores de índole económica y política, pero también los condicionantes culturales, como la necesidad de pertenencia y de sentirse "culturalmente 'en casa'", tal como expresó Wimmer (2013, p. 5), pueden ser clave en este proceso. Finalmente, la dignidad pisoteada, la falta de reconocimiento social, el estigma, la búsqueda de prestigio, pueden desencadenar la construcción de pertenencias grupales firmes.

\section{Anti-esencialismo analítico y esencialismos cotidianos}

Conceptos como "grupo", "sociedad" o "identidad" traen consigo una carga esencialista. La crítica de su uso se vincula fuertemente con el giro postmodernista, si bien en el ámbito que nos ocupa se ha insistido en el carácter cambiante y construido de la identidad, incluso antes del desarrollo de esta corriente de pensamiento (Jenkins, 2008). La visión de los grupos humanos como colectivos internamente homogéneos que tienen objetivos compartidos, son delimitados por fronteras claras y fijas y se arraigan en diferencias culturales tajantes requería de una revisión fundamental. Obras como "Orientalismo", de Said (2008 [1978]), han sido clave en el cuestionamiento del imaginario colonial sobre el "Otro" basado en el etnocentrismo Occidental. Con el tiempo, la deconstrucción de términos como "cultura" (Kuper, 2001), "etnicidad" o "identidad" (Brubaker; Cooper, 2001) permitió desvelar los problemas asociados al uso 
acrítico de estos conceptos. No deberíamos partir de las visiones esencialistas de la realidad social, pero ¿cómo abordar las percepciones esencialistas y sus repercusiones mostradas por diferentes actores sociales? Los discursos esencialistas constituyen un fenómeno real que puede influir en las pautas de comportamiento. Tal y como afirma Grillo (2003, p. 165), “teóricamente, la diversidad cultural no puede ser reducida a entidades inconmensurables y homogéneas (...) en la práctica esto tiene lugar constantemente". Las visiones esencialistas de la realidad social no aparecen sólo en discursos que podríamos clasificar como xenófobos o racistas, sino que constituyen un elemento presente en múltiples tipos de contextos e interacciones. En esta línea, Friedman (2002, p. 30) se pregunta: "si las personas se involucran en lo que se denomina creación de fronteras, cierre y esencialismo, ¿estos no deben ser reconocidos como fenómenos sociales reales en lugar de ser rechazados como errores terribles?".

En el ámbito de estudio de las categorías étnicas, nacionales y raciales, deberíamos enfatizar que las etiquetas identitarias constituyen un producto real de los procesos de categorización y pueden vincularse además con pautas de comportamientos particulares y diferenciados. Por esta razón Levine (1999, p. 165) abogó por la construcción de planteamientos teóricos que permitirían evitar los problemas del "positivismo ingenuo", pero también del "exceso nihilista de deconstruccionismo". En este sentido, Fenton (2003, p. 16), al abordar el tema de los grupos étnicos, indica que "sería un error afirmar que 'allí no hay nada', ya que sin duda sí, hay. Pero al mismo tiempo sería un error pensar que los 'grupos' son unos evidentes colectivos de personas (...) delimitados mediante unas líneas claras". Como apuntan Gupta y Ferguson (1992), las "comunidades imaginadas" (imagined communities) no son, en ningún caso, imaginarias (imaginary). El clásico texto de Anderson (1993) sobre los fundamentos del nacionalismo moderno muestra cómo puede ser que los individuos que no se conocen en persona pueden llegar a verse como componentes de una misma nación. El término "comunidades imaginadas" no hace referencia a realidades falsas, sino a un fenómeno social que puede adquirir una relevancia fundamental en la 
vida cotidiana. Así, los grupos humanos llegan a plasmarse en realidades tangibles a raíz de su involucración en procesos de categorización. Es necesario, por tanto, siguiendo las ideas de Riccio (2004), partir del antiesencialismo analítico sin perder de vista el impacto del esencialismo en los discursos y prácticas de las poblaciones que estudiamos.

Pero, ¿cómo pueden articularse las relaciones entre las categorías identitarias existentes y las pautas sociales? Se trata de una de las preguntas más importantes en el análisis del impacto de los procesos de identificación. A la hora de abordar la relación entre las etiquetas externas que se atribuyen a grupos específicos y su comportamiento real hay que tener en cuenta las siguientes cuestiones: i) diversidad intragrupal; ii) transformaciones de las pautas socio-culturales en el tiempo; iii) la frecuente falta de relación entre las etiquetas existentes y la realidad social; iv) la influencia de la discriminación en el surgimiento de algunos rasgos distintivos; y v) la sobreestimación de rasgos culturales por encima de los de índole política y económica. Veamos cada uno de estos puntos en detalle.

En primer lugar, hay que tener en cuenta la diversidad intragrupal, tanto en cuanto a las formas de categorización de la realidad social como en cuanto a la conducta. Se trata de una tarea difícil porque el proceso de categorización se asocia con los "efectos de acentuación" descritos por Tajfel (1984). Se trata aquí de la tendencia a exagerar la similitud de los componentes de una categoría y la diferencia entre categorías. Tal y como afirma García (2007, p. 51): “los mecanismos de construcción discursiva de lo colectivo son bastante simples: anulan diferencias internas y generalizan rasgos supuestamente distintivos". Como consecuencia, la diversidad interna se ve invisiblizada.

Especialmente desde la década de los 70, se consolida la visión de que la cultura es construida, mutable y profundamente contextual. Los individuos que comparten una misma categoría identitaria no necesariamente son portadores de una misma cultura. Las propias etiquetas con las que nos identificamos pueden significar cosas muy distintas para personas diferentes. Los individuos que se autodefinen como andaluces, estadounidenses, 
mujeres o activistas, pueden entender estas mismas categorías de maneras profundamente heterogéneas.

Las categorías identitarias se asocian con marcadores identitarios que sirven para delimitar fronteras sociales indicando quién pertenece al grupo y quién no. El clásico texto de Barth (1976) Ilamó la atención de la comunidad científica sobre esta realidad centrándose en los grupos étnicos y defendiendo que estos no equivalen a culturas particulares. Las fronteras entre grupos no se establecen en función de una simple suma de similitudes y diferencias culturales sino mediante los elementos que los actores sociales ven como relevantes. De esta forma, solo algunos elementos de un amplio espectro de pautas culturales son utilizados para definir las categorías identitarias. Esto tiene implicaciones fundamentales para la investigación. Ya no podemos afirmar que un grupo " $\mathrm{A}$ " es " $\mathrm{A}$ " porque habla un idioma " $\mathrm{A}$ " o práctica la religión " $\mathrm{A}$ ". Se defiende que deberíamos mirar, más bien, qué elementos de la realidad los miembros del grupo " $\mathrm{A}$ " consideran que les diferencian del grupo "B". En otras palabras, se debe indagar en cómo " $\mathrm{A}$ " sabe quién es "A" y quién es "B" y cómo construye la frontera entre ambos (Fenton, 2003). Los marcadores identitarios son los elementos de la realidad, como vestimenta, lengua, símbolos religiosos, etc., que los individuos utilizan, de manera consciente o inconsciente, como una herramienta para delimitar los grupos.

Las ideas de Barth no implican que la cultura no tenga importancia o que no existan diferencias culturales en normas, valores, costumbres, etc. que se puedan detectar a nivel colectivo. Enfatizar la importancia de la diversidad no significa que se ignoren rasgos compartidos socialmente en el seno de grupos humanos. Sin embargo, la identidad social no se fundamenta de manera directa en una cultura común, sino en la práctica de la categorización, tanto interna como externa. Los grupos se delimitan en función de un limitado número de elementos, que son utilizados para marcar la frontera y determinar quién pertenece al grupo y quién no. En este proceso, los marcadores identitarios, tratados como criterios de inclusión 
y exclusión, sirven para invisibilizar la diversidad interna y acentuar las diferencias entre el grupo propio y los demás.

La limitada capacidad cognitiva del ser humano, según Hale (2004), influye en que los marcadores identitarios suelen ser los elementos más simples y visibles, como las diferencias lingüísticas, fenotípicas o de vestimenta. A estos elementos se asocian otras características que, en su totalidad, forman una imagen prototípica de los miembros de una categoría específica.

La participación en un mismo dominio simbólico y la autodefinición grupal mediante la misma categoría identitaria puede ir acompañada por conflictos, tensiones internas en cuanto a los valores y normas, así como por inconformismo en cuanto al comportamiento. Las diferencias existentes suelen sin embargo ser empujadas a un segundo plano por los discursos que insisten en la unidad y la similitud. Por ello, las personas que, juzgando por las categorías identitarias, "son" iguales, no necesariamente "hacen" lo mismo (Jenkins, 2008). Es más, el uso de las mismas categorías identitarias durante prolongados periodos de tiempo no es óbice para que los significados atribuidos a ellas sean cambiantes. La comprensión de este mecanismo permitió entender cómo puede ser que ciertas categorías identitarias se mantengan en el tiempo, pese a las transformaciones de las pautas de comportamiento, cambios de membrecía o diversificación interna (Glazer; Moynihan, 1963).

Las categorías identitarias pueden ser comparadas con un paraguas que esconde tanto la heterogeneidad cultural como los conflictos identitarios. Por esta razón, el estudio de cada grupo debería verse acompañado de un análisis detallado de la diversidad y los procesos de negociación asociados a las pautas de comportamiento supuestamente compartidos.

En segundo lugar, hay que insistir en la importancia de las transformaciones de las pautas culturales en el tiempo en el caso de grupos etiquetados con una misma categoría identitaria. Esta precaución se asocia con la idea de que los grupos humanos no deberían ser tratados como entes estáticos. Como indica de forma expresiva Baumann (1999, p. 26), en la actualidad 
dejamos de ver la cultura como una "fotocopiadora", que convierte a las personas en reflejos pasivos de la realidad social, y adoptamos una perspectiva procesual en la que la cultura puede ser más bien comparada con una "históricamente improvisada jam session". Así, a pesar de la continuidad en el uso de una misma categoría identitaria, los patrones de comportamiento asociados a estas pueden transformarse en el tiempo.

Estas ideas son especialmente relevantes en el caso del análisis de las categorías identitarias de alta relevancia social. Las categorías de este tipo no constituyen un rasgo intrínseco de grupos humanos sino que se construyen en contextos particulares. Si nos encontramos con el desarrollo del conexionismo relacional de carácter exclusivista, la segregación residencial o el favorecimiento de los miembros del endogrupo en los procesos de contratación, etc., lo más probable es que estemos presenciando el afianzamiento de las fronteras sociales.

Además, los individuos tienen la capacidad de reconfigurar las categorías identitarias en las que fueron socializados. Existen evidencias empíricas que muestran, por ejemplo, que las migraciones pueden implicar la renegociación de los referentes identitarios. La movilidad de personas altamente cualificadas puede llevar, entre otros, al aumento de la importancia de las categorías identitarias vinculadas con la profesión (Colic-Peisker, 2010) o, en el contexto de la migración intra-UE, a una mayor identificación como europeos (Koikkalainen, 2013). En otros casos, la exclusión social en el contexto de destino puede implicar el fomento de la identificación con el origen (Aranda, 2007).

A veces la redefinición de las categorías identitarias tiene lugar a nivel colectivo. Sirva de ejemplo, el movimiento "Red power", que pretende invertir la imagen de las poblaciones indígenas de América (Wimmer, 2008a). Cuestionando el imaginario estigmatizador, el movimiento se propuso el uso del término "Primeras Naciones" para reinterpretar las percepciones predominantes sobre los indígenas. En los movimientos ligados a esta visión, se enfatiza el orgullo del patrimonio de estos grupos y se reivindica la herencia de su lucha, que sigue siendo invisibilizada 
en las cartas de la historia escrita por los conquistadores, desafiando las representaciones coloniales que muestran este patrimonio como atrasado y primitivo. Se reviven, o más bien (re)inventan (Hobsbawm, 2008) las tradiciones, como por ejemplo la de pow wow, olvidadas en el contexto de imposición cultural externa.

En tercer lugar, con frecuencia nos encontramos con la falta de consonancia entre etiquetas externas y las pautas de comportamiento de grupos identificados como "diferentes". Los grupos identificados como "otros" no tienen por qué mostrar ni las acciones ni las características que se les atribuye.

El concepto de identidad es utilizado con frecuencia en los discursos políticos para convencer a los individuos de que son similares entre sí y, a la vez, diferentes de los demás. Se promueve la idea de que se comparte la misma historia, intereses comunes y el mismo futuro. Se pretende así canalizar la acción siguiendo las líneas divisorias que surgen a nivel colectivo. Teniendo en cuenta esta realidad, Brubaker y Cooper (2001) indican que se pueden analizar los discursos nacionalistas o racistas sin asumir que los grupos descritos en estos discursos hacen referencia a grupos reales que unen a las personas que piensan y actúan de modo parecido.

Pensemos sobre el caso de las representaciones de la población judía durante la II Guerra Mundial. Por ejemplo, en Cracovia (Polonia), bajo la ocupación de los nazis, la ciudad fue inundada con propaganda antisemita, con carteles, folletos y exposiciones que mostraban a los judíos como el grupo que amenaza la humanidad. En el espacio urbano aparecieron eslóganes como "la nación judía de parásitos" o "la influencia corrumpente de los judíos". Las imágenes con individuos sucios y con muestras de enfermedades graves fueron utilizadas para convencer a la población general de que los judíos son responsables de la transmisión de patologías (Jodłowiec-Dziedzic, 2003). Se trata aquí de una ilustración sobre cómo los actores externos pueden llegar a construir una imagen, en base a información falsa que no tiene por qué ser reflejo de la realidad. Otro ejemplo, más reciente, hace referencia a las migraciones de los polacos y rumanos al Oeste de Europa. El 
texto de Sobis, Junjan y Vries (2016) analiza cómo esta movilidad se asocia con el surgimiento de discursos que exageran la migración real y que, a la vez, promueven mitos para proteger los intereses nacionales.

En cuarto lugar, deberíamos tener en cuenta la influencia de la discriminación en el surgimiento de algunos rasgos distintivos de poblaciones vistas como diferentes. Empecemos con una reflexión sobre cómo estos rasgos distintivos pueden plasmarse a nivel de la auto-identificación personal y colectiva para pasar, más adelante, a la relación entre la discriminación y el surgimiento de pautas distintivas en la conducta.

Las identificaciones por parte de actores sociales externos pueden estimular procesos identitarios grupales, sobre todo si nos encontramos con pautas que contribuyen a la exclusión social. En este sentido, es necesario recurrir al análisis de las relaciones de poder identificando a los actores sociales que cuentan con una mayor probabilidad de imponer sus categorizaciones sobre los demás. Si un grupo es visto como diferente y es tratado como tal, es probable que las categorías externas que generan esta realidad se vuelvan relevantes para los afectados. Especialmente el sentimiento de inseguridad, de ser amenazado desde fuera o estar en peligro, tiende a producir solidaridad intragrupal. Si las amenazas o ataques desde fuera se articulan en torno a categorías identitarias, la importancia de estas, pese a los conflictos en cuanto a su significado, puede aumentar tanto para los que identifican como para los identificados. En tales contextos se ha documentado la existencia de una mayor preponderancia de la aceptación del liderazgo centralizado y de la atribución de un alto valor a los símbolos de pertenencia al grupo. La inseguridad puede provocar el cierre social, percibido este como una forma de proteger a los suyos frente a las amenazas externas. Así, es fácil convertirse de una víctima de la xenofobia en un xenófobo (Maalouf, 1999). En estos casos se observa, siguiendo las palabras de Castells (2004), "la exclusión de los que excluyen por los excluidos".

En definitiva, la identidad personal se ve afectada por condicionantes externas que pueden limitar la libertad en la construcción de la autodefinición 
personal. A la vez el aumento de la relevancia de las categorías identitarias grupales puede generar patrones de comportamiento que refuerzan las divisiones entre grupos, sobre todo si nos encontramos con la discriminación. Las limitaciones impuestas desde fuera en estos casos pueden canalizar el desarrollo de formas de comportamiento compartidas.

Los estudios migratorios aportan evidencias que apoyan las ideas descritas. La instalación en un nuevo lugar a raíz de la movilidad puede ser acompañada por el descubrimiento de imaginarios preexistentes sobre la población migrante de diversos orígenes. Idioma, nombres o rasgos fenotípicos prevalentes en el origen pueden convertirse, en el destino, en marcadores identitarios que estigmatizan a la población migrante. Incluso las propias prácticas de etiquetaje por parte de la administración estatal pueden influir en que las categorías identitarias vinculadas con el origen sean fuertemente sentidas por parte de los migrantes. Pese a las previsiones de pensadores como Weber, los actuales estados liberales siguen estando étnicamente cargados (Kymlicka, 2010) y esta realidad tiene sus consecuencias en las relaciones con la población migrante. La definición de migrantes como "extranjeros" se asocia, ya de entrada, con derechos y obligaciones que les separan de los nacionales. Los procedimientos burocráticos a nivel estatal recuerdan de manera constante esta división, por lo que no es de extrañar que muchas personas acaben atribuyendo relevancia a las categorías identitarias vinculadas con su situación administrativa.

La pregunta fundamental es, sin embargo, cómo estas identificaciones externas pueden plasmarse en el comportamiento. En la literatura existente podemos encontrar múltiples ejemplos, que pueden servir para entender cómo una pauta de comportamiento diferenciada respecto al resto de la población puede surgir a raíz de los patrones de discriminación.

Sirva de ilustración el estudio de Bertrand y Mullainathan (2003), que desvela información valiosa para la comprensión de la inserción desventajosa de la población afroamericana en el mercado laboral de Estados Unidos. Los afroamericanos tienen efectivamente una mayor probabilidad de ocupar puestos de trabajo de más baja remuneración, menor prestigio 
social y más limitadas posibilidades de ascenso que el resto de la población estadounidense. Para indagar en las posibles causas de esta realidad, Bertrand y Mullainathan mandaron solicitudes respondiendo a ofertas de empleo en Boston y Chicago. Prepararon dos conjuntos de solicitudes. Los dos hacían referencia a candidatos ficticios con cualificaciones equivalentes y que, por tanto, deberían tener la misma oportunidad de conseguir trabajo. La única diferencia entre los dos conjuntos de solicitudes fueron los nombres de los candidatos. En un caso se asignaron nombres frecuentes en la población blanca, mientras que, en el otro, nombres más comunes entre los afroamericanos. Según los resultados, los candidatos con "nombres blancos" recibieron un 50\% más de invitaciones a entrevistas de trabajo que los candidatos con "nombres afroamericanos".

Podemos encontrar investigaciones que apuntan en la misma dirección en el caso de la segregación residencial. Los procesos de categorización pueden asociarse de manera pronunciada con la existencia de "enclaves étnicos" (Logan; Zhang; Alba, 2002). En el ámbito migratorio existen evidencias empíricas que muestran la relevancia de este fenómeno en múltiples contextos socio-geográficos e históricos (Thomas; Znaniecki, 2004 [1918-20]). El estudio de Checa y Arjona (2007) muestra esta realidad en el caso de la provincia de Almería. La segregación residencial en este contexto particular tiene que ver, según los resultados de la investigación, con el favorecimiento del endogrupo por parte de los nacionales. Se trata de una forma sutil de discriminación, pero que llega a tener un impacto muy pronunciado en las pautas de distribución geográfica de la población, llevando al desarrollo de un mercado inmobiliario dual. El origen de los clientes condiciona las estrategias de alquiler y de venta por parte de los propietarios, generando un submercado con una oferta limitada a zonas de escasa demanda y propiedades en precarias condiciones. Las personas de origen africano se ven especialmente afectadas por esta realidad. Las preferencias de los arrendadores reducen el número de propiedades disponibles para los migrantes, lo que, en un contexto de alta demanda, sirve para incrementar el precio de los alojamientos ofertados a ellos. 
Nos encontramos, en los ejemplos citados, con realidades que podrían ser interpretadas de una manera que culpabiliza a los propios migrantes. Sin embargo, en ningún caso, se debe pasar por alto el tema de la discriminación y de otros limitantes estructurales que pueden restringir la elección del lugar de asentamiento.

Patrones analógicos pueden ser encontrados en el caso de otros tipos de categorías identitarias, no necesariamente vinculadas con el origen. Sirva de ejemplo el texto de Tobio (2012), que aborda los factores que intervienen en la persistencia de la desigualdad en el reparto de las tareas de cuidado doméstico. Sí, es cierto que las mujeres siguen asumiendo una mayor carga de trabajos realizados en el ámbito doméstico. Sin embargo, en el análisis de esta realidad, no podemos dejar fuera el impacto de las prácticas de discriminación. Las diferenciadas pautas de comportamiento, según la autora, tienen que ver, entre otros, con las formas de construcción de la masculinidad, que siguen siendo impregnadas por ideas y relaciones patriarcales, desembocando en la frecuente reticencia masculina a asumir las obligaciones domésticas.

La última precaución se vincula, de manera estrecha, con el punto anterior. Argumentamos aquí que frecuentemente se sobreestima la relevancia de los rasgos culturales, restando importancia a los factores políticos y económicos.

Siguiendo las palabras de Anthias (2001), el debate académico y público puede acabar "atrapado en la cultura", obviando las realidades que tienen que ver con las desigualdades sociales y los patrones de exclusión social. Sirva de ejemplo el caso de las poblaciones musulmanas en Europa Occidental. A raíz de los procesos de categorización, se creó lo que Jeldtoft (2014) denomina el islam "hipervisible". Se trata de un imaginario que reduce el debate sobre la población musulmana a cuestiones como el radicalismo, terrorismo o expresiones de la religión en los espacios públicos. Se aborda el tema de la diversidad cultural partiendo de la idea de que existen diferencias fundamentales en cuanto a valores, creencias, costumbres y tradiciones. Si bien las diferencias culturales pueden ser relevantes a la 
hora de analizar las relaciones entre grupos, el análisis de la construcción de categorías identitarias no debe perder de vista el amplio panorama de las desigualdades socioeconómicas y políticas, que pueden convertir el ámbito identitario en un área de coerción pero también de resistencia y contestación.

\section{Conclusiones}

La identidad personal es multidimensional y está formada por una amplia variedad de puntos de referencia, aunque existan discursos que pretendan mostrarla como algo reducido a una única pertenencia, sea esta de carácter étnico, religioso, racial o de otro tipo. Esta multidimensionalidad, entre otros factores, influye en que los colectivos humanos no sean homogéneos internamente. Si conceptualizamos la identidad personal como un conjunto de puntos de referencia vinculados entre sí, que además pueden cambiar en cuanto a su significado y relevancia, el riesgo de esencializar a los grupos humanos se ve disminuido.

La investigación en ciencias sociales y humanidades debe partir de una necesaria sensibilidad analítica a la diversidad cultural y a los procesos de cambio. Sin embargo, esto no puede hacernos perder de vista la gran influencia que el pensamiento esencialista puede ejercer en los procesos de categorización. Argumentamos, por tanto, que uno de los problemas fundamentales en el estudio del ámbito identitario tiene que ver con la insuficiente comprensión de los esencialismos cotidianos.

En la realidad social se pueden observar patrones y regularidades que generan expectativas en cuanto al comportamiento de los demás, orientando así nuestros propios actos. El proceso de categorización es, hasta cierto punto, inevitable. Sin embargo, no debemos ver los grupos humanos como portadores de culturas e identidades radicalmente diferentes entre sí. Realizar este tipo de equiparaciones es tan simplista que deja de ser verdadero. Tanto en los discursos académicos como públicos utilizamos diferentes tipos de categorías identitarias, como "franceses", "migrantes", "budistas", 
"hombres", "trabajadores" etc. Existen dos vías principales de cuestionar la carga esencialista que subyace en cada uno de estos términos. Una consiste en abandonar por completo los términos étnicos, nacionales, religiosos etc. como categorías analíticas. La segunda se basa en el uso de estos términos pero teniendo en cuenta una serie de precauciones fundamentales. Conceptualizar la identidad como un fenómeno multidimensional permite apreciar la heterogeneidad ligada a cuestiones como la estructura de edad, la desigualdad de oportunidades educativas, las diferenciadas trayectorias laborales, la diversidad de creencias etc. Las categorías identitarias, así como las pautas de comportamiento que pueden asociarse a estas, están sujetas a procesos de negociación en cuanto a su significado y relevancia, tanto a nivel personal como colectivo. Dicha negociación queda fuertemente marcada por el diferenciado acceso al poder, recursos y oportunidades tanto entre grupos como en el seno de estos. Pero la pluralidad interna y la variabilidad en el tiempo no son todo, ya que, con frecuencia, existe una ausencia absoluta o casi absoluta de relación entre las categorizaciones y el comportamiento o características reales de los grupos etiquetados como "diferentes". Finalmente, la comprensión de la diversidad identitaria y cultural no debe pasar por alto el hecho de que detrás de algunos rasgos distintivos pueden encontrarse patrones de discriminación y profundas desigualdades socioeconómicas y políticas.

Rita Sobczyk es socióloga y antropóloga con especialización en estudios migratorios y profesora en el Departamento de Historia y Ciencias Sociales de la Universidad del Norte, Barranquilla, Colombia.

凶rsobczyk@uninorte.edu.co

Rosa Soriano-Miras es Doctora en Sociología y profesora titular del Departamento de Sociología de la Universidad de Granada, Granada, España.

$\triangle$ rsoriano@ugr.es

Andrés Caballero-Calvo es Doctor en Geografía y Desarrollo Territorial y profesor en el Departamento de Ciencias Sociales de la Universidad del Norte, Barranquilla, Colombia.

$\triangle$ andrescaballero@uninorte.edu.co 


\section{Referencias}

1. ALBA, Richard. Bright vs. blurred boundaries: second-generation assimilation and exclusion in France, Germany, and the United States. Ethnic and Racial Studies, v. 28, n. 1, p. 20-49, 2005.

2. ANDERSON, Benedict. Comunidades imaginadas. Reflexiones sobre el surgimiento y difusión del nacionalismo. Ciudad de México: Fondo de Cultura Económica, 1993.

3. ANTHIAS, Floya. New hybridities, old concepts: the limits of "culture". Ethnic and Racial Studies, v. 24, n. 4, p. 619-641, 2001.

4. ARANDA, Elizabeth. Struggles of incorporation among the Puerto Rican middle class. Sociological Quarterly, v. 48, n. 2, p. 199-228, 2007.

5. BARTH, Fredrik. Los grupos étnicos y sus fronteras: la organización social de las diferencias sociales. Ciudad de México: Fondo de Cultura Económica, 1976.

6. BAUMAN, Zygmunt. Identity: conversations with Benedetto Vecchi. Londres: John Wiley \& Sons, 2013.

7. BAUMANN, Gerd. The multicultural riddle: rethinking national, ethnic, and religious identities. Nueva York: Psychology Press, 1999.

8. BAUMANN, Gerd; GINGRICH, Andre. Grammars of identity/alterity: a structural approach. Nueva York: Berghahn Books, 2006.

9. BERTRAND, Marianne; MULLAINATHAN, Sendhil. Are Emily and Greg more employable than Lakisha and Jamal? A field experiment on labor market discrimination. American Economic Review, v. 94, n. 4, p. 991-1013, 2003.

10. BREWER, Marilynn; SILVER, Michael. Group distinctiveness, social identification, and collective mobilization. In: STRYKER, Sheldon; OWENS, Timothy J.; WHITE, Robert W. (Eds). Self, identity, and social movements, 2000. p. 153-171.

11. BRUBAKER, Rogers. Ethnicity without groups. Archives Européennes de Sociologie, v. 43, n. 2, p. 163-189, 2002.

12. BRUBAKER, Rogers. The "diaspora" diaspora. Ethnic and Racial Studies, v. 28, n. 1, p. 1-19, 2005.

13. BRUBAKER, Rogers. Ethnicity, race, and nationalism. Annual Review of Sociology, v. 35, n. 1, p. 21-42, 2009.

14. BRUBAKER, Rogers. Categories of analysis and categories of practice: a note on the study of Muslims in European countries of immigration. Ethnic and Racial Studies, v. 36, n. 1, p. 1-8, 2012.

15. BRUBAKER, Rogers; COOPER, Frederick. Más allá de la "identidad". Apuntes de investigación del CECYP, n. 7, p. 1-66, 2001. 
16. BRUBAKER, Rogers; LOVEMAN, Mara; STAMATOV, Peter. Ethnicity as cognition. Theory and Society, v. 33, n. 1, p. 31-64, 2004.

17. CASTELLS, Manuel. The power of identity: the information age - economy, society, and culture. Oxford: Blackwell Publishing, 2004.

18. CHECA, Juan C.; ARJONA, Ángeles. Factores explicativos de la segregación residencial de los inmigrantes en Almería. Revista Internacional de Sociología, v. 65, n. 48, p. 173-200, 2007.

19. COLIC-PEISKER, Val. Free floating in the cosmopolis? Exploring the identitybelonging of transnational knowledge workers. Global Networks, v. 10, n. 4, p. 467-488, 2010.

20. CSEDŐ, Krisztina. Markets and networks: channels towards the employment of Eastern European professionals and graduates in London. In: OKOLSKI, M.; BLACK, R.; ENGBERSEN, G. (Eds.). A continent moving west? Amsterdam: IMISCOE, 2010. p. 89-111.

21. ESCOBAR, Modesto; REVILLA, Juan Carlos; SÁNCHEZ-SIERRA, Marina. La influencia de la situación y el entrevistador en la expresión de la identidad personal. Revista Internacional de Sociología, v. 76, n. 3, p. 1-15, 2018.

22. FENTON, Steve. Ethnicity. Cambridge: Polity Press, 2003.

23. FRIEDMAN, Jonathan. From roots to routes: tropes for trippers. Anthropological Theory, v. 2, n. 1, p. 21-36, 2002.

24. GARCÍA, José L. Cultura. In: BARAÑANO, A.; DEVILLARD, M.; CÁTEDRA, M.; GARCÍA, J. L. (Eds.). Diccionario de relaciones interculturales: diversidad y globalización. Madrid: Editorial Complutense, 2007. p. 47-51.

25. GIDDENS, Anthony. Modernity and self-identity. Cambridge: Polity, 1991.

26. GLAZER, Nathan; MOYNIHAN, Daniel. Beyond the melting pot: the Negroes, Puerto Ricans, Jews, Italians and Irish of New York City. Cambridge: MIT Press, 1963.

27. GOFFMAN, Erving. La presentación de la persona en la vida cotidiana. Buenos Aires: Amorrortu, 2001.

28. GÓMEZ, Angel; VÁZQUEZ, Alexandra. Identidad personal e identidad social ¿Dos procesos diferentes o uno solo? Revista de Psicología Social, v. 30, n. 3, p. 474-480, 2015.

29. GRILLO, Ralph. Cultural essentialism and cultural anxiety. Anthropological Theory, v. 3, n. 2, p. 157-173, 2003.

30. GUPTA, Akhil; FERGUSON, James. Beyond "culture": space, identity, and the politics of difference. Cultural Anthropology, v. 7, n. 1, p. 6-23, 1992.

31. HALE, Henry. Explaining ethnicity. Comparative Political Studies, v. 37, n. 4, p. 458-485, 2004. 
32. HALE, Henry; SHEVEL, Oxana; ONUCH, Olga. Believing facts in the fog of war: identity, media and hot cognition in Ukraine's 2014 Odesa tragedy. Geopolitics, v. 2, p. 1-31, 2018.

33. HALL, Stuart. Diaspora and visual culture. Londres: Routledge, 2014.

34. HOBSBAWM, Eric. Wynajdywanie tradycji. Cracovia: UJ, 2008.

35. JELDTOFT, Nadia. The hypervisibility of Islam. In: WOODHEAD, L.; DESSING, N. M.; NIELSEN, J. S. (Eds.). Everyday lived Islam in Europe. Aldershot: Ashgate Publishing, 2014.

36. JENKINS, Richard. Social identity. Nueva York: Routledge, 2008.

37. JODŁOWIEC-DZIEDZIC, Anna. The holocaust of Cracow's Jews. Cracovia: The Historical Museum of the City of Cracow, 2003.

38. KOIKKALAINEN, Saara. Transnational highly skilled Finnish migrants in Europe: choosing one's identity. National Identities, v. 15, n. 1, p. 85-100, 2013.

39. KUPER, Adam. Cultura: la versión de los antropólogos. Barcelona: Paidós, 2001.

40. KYMLICKA, Will. Ethnicity and liberalism in the USA. In: REX, J.; GUIBERNAU, M. (Eds.). The ethnicity reader: nationalism, multiculturalism, and migration. Cambridge: Polity Press, 2010. p. 255-272.

41. LAMONT, Michèle; MOLNÁR, Virág. The study of boundaries in the Social Sciences. Annual Review of Sociology, v. 28, n. 1, p. 167-195, 2002.

42. LEVINE, Hal. Reconstructing ethnicity. Journal of the Royal Anthropological Institute, v. 5, p. 165-180, 1999.

43. LOGAN, John; ZHANG, Wenquan; ALBA, Richard. Immigrant enclaves and ethnic communities in New York and Los Angeles. American Sociological Review, v. 67, n. 2, p. 299-322, 2002.

44. MAALOUF, Amin. Identidades asesinas. Madrid: Alianza, 1999.

45. MEAD, George H. Mind, self, and society: from the standpoint of a social behaviorist. Chicago: University of Chicago Press, 2009.

46. OLMOS, Antonia. La población inmigrante extranjera y la construcción de la diferencia: discursos de alteridad en el sistema educativo andaluz. Granada: Universidad de Granada, 2009.

47. PEEL, Daniel. The cultural work of Yoruba ethnogenesis. In: HUTCHINSON, J.; SMITH, A. (Eds.). Nationalism: critical concepts in political science. Nueva York: Routledge, 2000. cap. 13.

48. RICCIO, Bruno. Transnational Mouridism and the Afro-Muslim critique of Italy. Journal of Ethnic and Migration Studies, v. 30, n. 5, p. 929-944, 2004.

49. SAID, Edward. Orientalismo. Barcelona: Debolsillo, 2008. 
50. SOBIS, Iwona; JUNJAN, Veronica; VRIES, Michiel. Polish plumbers and Romanian strawberry pickers: how the populist framing of EU migration impacts national policies. Migration and Development, v. 5, n. 3, p. 431-454, 2016.

51. STETS, Jan; BURKE, Peter. Identity theory and social identity theory. Social Psychology Quarterly, v. 63, n. 3, p. 224-237, 2000.

52. TAJFEL, Henri. Grupos humanos y categorías sociales: estudios de psicología social. Barcelona: Herder, 1984.

53. THOMAS, William; ZNANIECKI, Florian. El campesino polaco en Europa y en América. Madrid: Centro de Investigaciones Sociológicas, 2004.

54. TILLY, Charles. Identities, boundaries and social ties. Londres: Routledge, 2015.

55. TOBíO, Constanza. Cuidado e identidad de género. De las madres que trabajan a los hombres que cuidan. Revista Internacional de Sociología, v. 70, n. 2, p. 399-422, 2012.

56. UVIN, Peter. Ethnicity and power in Burundi and Rwanda: different paths to mass violence. Comparative Politics, v. 31, n. 3, p. 253-271, 1999.

57. WIMMER, Andreas. Elementary strategies of ethnic boundary making. Ethnic and Racial Studies, v. 31, n. 6, p. 1025-1055, 2008a.

58. WIMMER, Andreas. The making and unmaking of ethnic boundaries: a multilevel process theory. American Journal of Sociology, v. 113, n. 4, p. 9701022, $2008 b$.

59. WIMMER, Andreas. Ethnic boundary making: institutions, power, networks. Oxford: Oxford University Press, 2013.

60. WIMMER, Andreas. Ethnic boundary making as strategic action: reply to my critics. Ethnic and Racial Studies, v. 37, n. 5, p. 834-842, 2014.

Recibido el: 5 jul. 2019.

Aprobado el: 4 mar. 2020. 